\title{
HACIA UNA VISIÓN DEL PROYECTO DE VIDA EN PERSONAS CON DISCAPACIDAD MENTAL
}

\author{
* Marta Lucía Santacruz González
}

El mundo está lleno de caras comprensibles; cuando uno nace, desde ese momento, se explica uno por qué y cuándo estamos aqui en este mundo, deseo o pienso o sea comprendo pido su teta a mama, le sonrío y le agradezco con un gesto de agradecimiento (Alberto Henao, junio 2003. Usuario UPA Servitá.)

\section{Resumen}

El presente artículo intenta una aproximación al tema, "proyecto de vida" en personas con discapacidad mental, el cual se construye desde la experiencia directa con este tipo de población y mediante un programa de 5 meses; esta vivencia se convierte en la tercera fase de un proceso desarrollado por la Facultad de Terapia Ocupacional de la Institución Universitaria Fundación Escuela Colombiana de Rehabilitación, dentro de los programas de participación comunitaria del Hospital de Usaquén E.S.E ( Empresa Social del Estado), entre enero de 2002 y junio de 2003, proyecto encaminado a trabajar la competencia ocupacional en estas personas. Involucra también acciones contextualizadas en lo que establece el Plan Distrital de Discapacidad 2001-2005 y el documento "Lineamientos de Atención en Salud para las Personas con Discapacidad en Bogotá", ambos de la Secretaria Distrital de Salud de Bogotá. Se presentan algunas explicaciones sobre concepto de vida, proyecto de vida y su relación con Terapia Ocupacional, además, la autora ofrece desde su experiencia, una descripción cualitativa fundamentada en el modelo de la Ocupación Humana y del Desempeño Ocupacional Realizante, sobre cómo se presenta la capacidad para diseñar el proyecto de vida en personas con enfermedad mental, y a partir de esto, se plantea desde el Enfoque de la Logoterapia, una propuesta para intervenir en este campo. Se aclara al lector, que dentro del discurso se entremezclan escritos de algunos usuarios sobre cada una de las temáticas trabajadas, tratando asi de humanizar el presente.

Palabras Clave: Salud Mental, Discapacidad Mental, Logoterapia, Proyecto de vida

* Terapeuta Ocupacional. Especialista en Docencia Universitaria. Docente Facultad de Terapia Ocupacional Institución Universitaria Fundación Escuela Colombiana de Rehabilitación 


\section{INTRODUCCIÓN}

L a temática presentada en este artículo surge del hecho que en Colombia el número de personas con discapacidad mental ha mostrado un incremento en los últimos años, situación que ha demandado por parte de los sectores públicos y privados, así como de las entidades que forman profesionales en el campo de la rehabilitación, acciones encaminadas a mejorar la calidad de vida de dicha población y sus familias, actuando en los niveles de prevención, promoción y rehabilitación de la salud mental de los Colombianos (Ministerio de Salud, 1998). En relación con esto, el Comité Técnico del Consejo Distrital para las personas con discapacidad en Bogotá, dentro de su Plan Distrital para la atención de las personas con discapacidad, contempla, además de otros principios de intervención basados en las necesidades de las personas con discapacidad y por ende de aquellas con enfermedad mental, el derecho a tener un proyecto de vida y mejorar su calidad de vida, en esto la educación al usuario y su familia son elementos fundamentales para obtener una vida independiente con un nivel de participación comunitaria que redunde en su bienestar, actuando a su vez, como factor preventivo de sus crisis y condiciones secundarias a su discapacidad.

De lo expuesto anteriormente, se generó el proyecto que ocupa este artículo, el cual se enmarcó en los delineamientos dados por la política en seguridad social y las que rigen la salud mental en Colombia, de manera específica se centró en las líneas de intervención que establece el Plan Distrital de Discapacidad 2001-2005, y en los lineamientos de atención en salud para las personas con discapacidad en Bogotá, donde se plantea el ciclo evolutivo, el proyecto de vida, el estatus de edad, el rol ocupacional, el entorno y las expectativas como elementos indispensables que tienen que ver con las acciones, intervenciones y decisiones que se realicen con las personas con discapacidad y sus familias. Partiendo de esta visión, la experiencia en mención se ubicó en el nivel de prevención terciaria, la que según Disability in American (Kniepman,1997), recomienda una aproximación comprensiva para la prevención de condiciones secundarias en personas con discapacidad y por otro lado, en la promoción como el fortalecimiento del funcionamiento positivo. Las acciones se diseñaron con base en el enfoque de Proyecto de Vida y el principio de calidad de vida que el Comité plantea en sus lineamientos, a través de un programa que articuló lo anterior, con la visión del Terapeuta Ocupacional en el campo de la Salud Mental Comunitaria y la corriente terapéutica de la Logoterapia.

Es así como esta experiencia psicoeducativa, se fundamentó en el modelo de la Ocupación Humana (Kielhofner, 1985), y del Desempeño Ocupacional Realizante (Trujillo, Alvarez, Torres y Zapata, 1992), desde los cuales se da una aproximación sobre la capacidad para diseñar y desarrollar el proyecto de vida en personas con discapacidad mental, y asi mismo se presenta desde el enfoque de la Logoterapia con estrategias de intervención mediante un programa que ha sido denominado "Proyecto de vida y competencia ocupacional en población adolescente y adulta con discapacidad mental " (Santacruz y Cols. 2003). Como ya se mencionó, el presente trabajo conforma el tercer módulo de un proceso psicoeducativo en población con discapacidad mental y cognoscitiva, llevado a cabo por la Facultad de Terapia Ocupacional de la Institución Universitaria Fundación Escuela Colombiana de Rehabilitación, el cual se inició en enero del 2002, donde la primera línea de trabajo se contextualizó en la estrategia de estilos de vida saludables y se denominó "promoción de patrones ocupacionales saludables" y la segunda fase se enmarcó en los derechos y deberes de las personas con discapacidad mental, titulada "Promoción del comportamiento ocupacional competente a partir del conocimiento de los derechos y deberes en población con discapacidad mental y cognoscitiva, dentro y fuera del entorno familiar"; ambos encaminados a facilitar la inclusión social de personas con discapacidad mental y cognoscitiva, y gestados a partir de las problemáticas que el Comité Técnico del Consejo Distrital para las personas con discapacidad identifica, y sobre las que establece en su plan de intervención una serie de estrategias 
encaminadas a recuperar su dignidad como personas parte de un colectivo. En relación con lo anterior, el Terapeuta Ocupacional como profesional con una aproximación humanista y holística del ser humano, cree que el sujeto con discapacidad tiene el potencial para apropiarse de su proceso de vida como proyecto y adquirir responsabilidad del mismo en la medida de sus habilidades remanentes. De manera específica, trabaja hacia la vida independiente de la persona con discapacidad manejando la ocupación como el eje centralizador y dinamizador de dichos procesos.

\section{Aproximación teórica al CONCEPTO "PROYECTO DE VIDA"}

Oportunidades y desbares el propósito de todos, la conquista del oeste unos mueren y otros triunfan en esta época yo existo es la diversión o la llamada libertad (Alberto 2003. Usuario UPA Servitá).

Abordar la temática sobre el Proyecto de Vida, hace necesario partir de la definición del concepto de Vida. Para algunos, la vida es un hecho evidente, un fenómeno de la naturaleza o el resultado de un movimiento cósmico que lleva implícito la ley de la evolución, o bien, la vida es el desarrollo y desenvolvimiento biológico de una entidad orgánica; de otro lado, la vida se considera el estado previo a la muerte, es decir tiene comienzo y fin, pues todo en la naturaleza tiene principio y fin, comienza con el nacimiento del individuo y termina con la muerte, como el fin de la vida del sujeto.

En este sentido, la ontología, que estudia al ser en cuanto Ser, considera la vida como el espacio de la existencia donde el individuo es, existe y trasciende, así, el existencialismo como corriente filosófica del siglo XX en la figura de Soren Kierkegaard (siglo XIX), concibe el desarrollo humano desde el significado de existencia, centrando su interés en problemas netamente humanos relacionados con la condición y naturaleza humana, como la soledad, el sufrimiento, la muerte, la incertidumbre, el dolor y la enfermedad. Para el existencialismo, lo primordial es la superación continua de sí mismo, en el sentido de posibilidad ontológica siempre nueva, y quien lo hace se proyecta en el futuro libremente, está siempre en sí mismo, en sus proyectos, esperanzas y temores. Esta manifestación de la existencia del ser humano, se inicia desde el momento en que toma conciencia sobre su existir en el mundo, de ahí que el fluir de la naturaleza humana se convierta en la dinámica de su existencia mediante cambios en la formación física, espiritual, moral, intelectual y psicológica, para lograr el máximo potencial de habilidades, destrezas y competencias; la construcción de la propia vida en relación permanente entre el mundo interno-subjetivo con el mundo externo-objetivo, da paso al ser íntegro, único, diferente y autónomo hasta lograr la trascendencia, (Franco Z.1994).

Para Inés Pardo, (1999) La dimensión humana se vislumbra desde la pirámide: sentir, pensar y actua, influenciada por factores que actúan dentro de una espiral: físicos, emocionales, familiares, laborales, educativos, culturales y sociales, que proyectados llevan a una vida eficiente llena de calidad, seguridad para afrontar lo nuevo y sobre lo que se estructura la capacidad productiva. Por su parte, el filósofo Federico Nietzche, afirma que el hombre posee voluntad de poder para realizarse relacionada con todas sus capacidades, facultades y posibilidades, que lo hace superior, dotado para determinar por sí mismo el bien y el mal, para crear sus propios valores, la felicidad, la paz, la construcción del propio proyecto vital en permanente interacción del cuerpo material con el alma espiritual. En este sentido, Fernando Savater en su libro diccionario Filosófico (1995), se refiere así a la condición humana : "somos seres naturales, dotados de instintos, de necesidades, de requisitos, amenazas y compensaciones fisiológicas; pero seres sociales también, formados en la imitación y celebración de lo humano, en el rechazo de la muerte, en la invención técnica, en la prótesis artificial que cumple o agiganta deseos naturales" pág. 140 .

Desde una visión oriental, el Dalai Lama en Aproximación Budista al Conocimiento de Matthew Bunson (1997), refiere que : " hemos sido bendecidos con la vida humana y todas las posibilidades que ello 
implica. Sin embargo la muerte nos presiona, amenazándonos con robarnos esta oportunidad preciosa en cualquier momento y cuando muramos sólo perdurarán las semillas de nuestro trabajo en la vida y de nuestro conocimiento espiritual. De ahí que el sentido personal de la vida se refiera a la interpretación, dirección u orientación que cada persona imprime a la ruta, a la trayectoria de su existencia y puede representar cualesquiera de los valores que se han asimilado durante la experiencia de vivir. Sentido que está dado por la proyección de cada individuo y se relaciona con trabajar, estudiar, divertirse, practicar deportes, entre otras actividades que partan de sus intereses y motivaciones. Es decir, para cada quien la vida tiene un sentido muy personal, pero el íntimo sentido de la existencia humana es el Saber, adquirir conocimiento, aprender, abrir una senda hacia la luz del entendimiento, es decir el Saber le da valor, consistencia y estímulo a todas nuestras acciones, estemos o no conscientes de ello" pág. 161.

Esta relación hombre-vida, la expresa también Jorge Yarce (2002), afirmando que la empresa de ser hombre es la más ambiciosa y difícil, pero la más necesaria en la vida de una persona. Sin ella no se es realmente persona, en cuanto que por el sólo hecho de venir al mundo o de crecer y desarrollarse físicamente no se tiene una personalidad, ésta se conquista y se realiza progresivamente y para lograrlo hay que estar constantemente haciéndose a sí mismo, lo que se recibe como legado y dependencia del entorno, no es tanto como lo que se puede hacer de cada uno libremente y se es feliz en la medida de querer y poder volver realidad lo que se espera de sí mismo. Es así como cada uno forja su propio proyecto de vida y lo saca adelante como cuando se esculpe una estatua, no sólo para admirarla, sino para sentirla, vivirla y encarnarla plenamente. El proyecto de vida se asocia a objetivos a corto, mediano y largo plazo, de esta manera se asocia a construcción, no es algo dado por herencia o transmisión en un individuo pasivo, se relaciona con el ir siendo. Así, una personalidad madura muestra un comportamiento y una vivencia laboral configurados bajo una modalidad adaptativa entre sus motivaciones y los valores del mundo ; de ahí la importancia de la identidad, conocer las motivaciones más profundas, la identidad de rol con las características que la personalidad va desarrollando desde los roles que desempeña.

Dichas motivaciones y la identidad, para Inés Pardo Barrios (1999), se configuran en el proyecto de vida, que ella define como tomar conciencia sobre las posibilidades de triunfar, reconocer las habilidades y debilidades, dirigir con autonomía el rumbo de la vida, enfocando la construcción del proyecto vital, esencial para salir adelante en el nicho afectivo y el círculo académico. Dentro del ciclo vital se reconoce que la vida va hacia adelante, que el tiempo es una circunstancia, es importante que el ser humano deje rastro y haga esfuerzo por vislumbrar un porvenir, lo que depende del empeño con que asuma el presente y la conciencia para elegir en pro de un mejor vivir. Este fin de vivir, lo presenta Savater (1995) determinado por dos grandes áreas que abarcan todas nuestras preferencias ideales, las que llama el área de la vida y el área de la libertad: “el área de la vida recoge las apetencias de salud, progenie, satisfacciones sensuales, aborrecimiento del dolor, seguridad y resguardo ante la hostilidad de la interperie, fuerza física y belleza entendida como apetecible aptitud para la tarea carnal, abundancia de proviciones, fidelidad a los más próximos y semejantes, ternura paternofilial, concordia comunitaria, compasión por los que sufren, contagio simpático con los que disfrutan. El área de la libertad congrega valores de excelencia y riesgo, el atrevimiento de la novedad, el reto a lo desconocido, el afán de conocimiento, la afirmación de la diferencia propia y la victoria sobre lo diferente, el anhelo de inmortalidad, la conquista, la invención, la institución de lo perdurable, la transgresión de los límites y las rutinas, la protesta ante lo obligado, la rebelión, la exigencia de perfección y justicia, la autonomía individual. Siendo evidente que los valores pertenecientes a cada área se intersectan, se impregnan y se refuerzan con frecuencia mutuamente" pág. 141. 
El proyecto de vida y los factores que lo cimentan, son argumentados desde una visión espiritual por Viktor Frankl (1990), creador de la Logoterapia, como una psicoterapia centrada en el sentido, cuyo argumento básico es que cada persona lucha para encontrar el sentido de su vida y trabaja la tendencia del hombre a la autorrealización, la cual se da en la medida que el hombre cumple el sentido y realiza sus valores. Es por tanto, una terapia que va desde el logos hacia lo espiritual (persona existencial) y busca la potenciación del sentido de vida, partiendo del sentido personal y concreto de la vida, toma la dimensión espiritual que aborda al hombre como un ser corporal, anímico y espiritual, ser espiritual que no está en el cuerpo, sino en todas partes y en ninguna. Para Frank1, la Logoterapia es el arte del encuentro interpersonal, en que la técnica se pone al servicio del que sufre, el paciente; refiere que el hombre es un ser que se encuentra en constante búsqueda del sentido, búsqueda del sentido que aparece en las circunstancias actuales de la sociedad y en las personas con una enfermedad mental, como un empeño vano y un vacio existencial, frustración que se debe en buena parte a la tríada que se compone de sufrimientos, culpa y muerte.

Las aproximaciones presentadas hasta aquí, contextualizan la tríada "ser humano -condición humanavida", en un proceso dinámico y continuo que cumple etapas y un propósito existencial que le da sentido al concepto de ser en el mundo, bajo principios de autoconciencia, libertad y autonomía, que se hacen palpables a través del acto, el hacer o la acción misma, sobre la cual se afianza el sentido existencial del Ser. Sentido que se hace visible en el propósito vital o bien en el proyecto de vida que cada individuo desde su unicidad elige, diseña, crea y comparte con el colectivo, reafirmando así su identidad y su estar en el mundo, más allá de una vivencia material, es decir alcanzar la trascendencia, que según Jaspers constituye por sí misma una esfera original, la esfera del Ser en sí . Igualmente, es un proceso continuo, "Proyecto de vida", conformado por diferentes elementos que podrán parecer abstractos, pero que indiscutiblemente le confieren sustento a lo que debe trabajar el individuo para lograr darle significado a su existencia y obtener un espacio en el tiempo, estos son: el sentido existencial, el sistema relacional yo-mundo, la identidad, el Yo Ideal, la autonomía, la toma de decisiones, el asumir actitudes con compromiso y la elaboración de metas personales, las cuales se definen en " Proyecto de vida y competencia ocupacional en población adolescente y adulta con discapacidad mental y cognoscitiva", Santacruz y Cols (2003).

De esta manera elaborar el proyecto de vida implica que se parta de la realidad propia y personal teniendo en cuenta:

- El ser, saber quién se es

- Cómo se es, es decir los rasgos de comportamiento, cualidades, defectos o bien habilidades y debilidades.

- Dónde se está, el entorno en que se desenvuelve el individuo.

- Con quién se vive, que incluye el núcleo familiar, amigos y parientes que de alguna manera influyen.

- Cómo se vive, es decir el estilo de vida

- Valores que determinan la vida, como los intereses hacia lo material, lo espiritual, el placer u otros.

Por otro lado, establecer pasos, partiendo de los objetivos trazados, concretar acciones y estrategias, referidas a :

- En la formación personal, el tiempo de estudio y trabajo

- En el tiempo libre, la cultura, el arte, el deporte u otras.

- En la relación familiar, sistema de comunicación e interacción, roles

- En la vida espiritual, bien sea la religión, voluntariado, altruismo u otras.

\section{EL PROYECTO DE VIDA DESDE TERAPIA OCUPACIONAL}

Me gustaría tener comprensión, para poder vivir fácilmente me gustaria que se terminara la guerra, para poder ponerme a disfrutar la vida eternamente 


\section{ARTÍCULOS INÉDITOS}

y terminar siendo un gran amigo, ya que tengo esa cualidad. Me gustaría que el niño Dios me volviera algo especial, como mi papá o lo que quiera. (Alberto, Usuario UPA Servitá, 2003).

Los baluartes para el proyecto de vida, son la calidad de los recursos dados por el entorno, los componentes motivacionales internos del individuo, la participación de factores existenciales como el sentido de sí mismo, el sentido de vida, la libertad, la autonomía, la capacidad productiva y la búsqueda de la autorrealización, ya planteados por diversos autores con diferente orientación conceptual, cualidades humanas esenciales en lo que concierne al proceso vital individual y sobre el que la persona descubre el significado de su existencia. Es así, que para el profesional de la ocupación humana, el hacer con sus diversas dimensiones y significados, actúa como el impulsor, organizador y representante del ser como proyecto y resultado; además el proyecto de vida demanda un individuo con historia y un ser en búsqueda de la autorrealización. De manera importante, Trujillo, Alvarez, Torres y Zapata (1992), en el denominado modelo del desempeño ocupacional realizante, afirman que "es mediante el actuar ocupacional que el ser humano tiende a su realización; desempeño ocupacional realizante que va más allá del actuar eficaz, apropiado y eficiente ; se trata de una trayectoria individual en la cual intervienen el potencial y capacidades sensoriomotoras, su cognición y sentimientos, las motivaciones, intereses, frustraciones y las metas de la vida" pág. 11 .

Sumado a lo anterior, para el Terapeuta Ocupacional, el proyecto de vida se relaciona de manera directa con una historia de vida, conformada a su vez por una historia personal donde las relaciones objetales y ambientales con su carga de símbolos idiosincráticos, sociales y universales, van configurando a través de la participación activa en ocupaciones significativas generadoras de placer y aprendizaje la historia ocupacional del sujeto, y por lo tanto su identidad como ser ocupacional con pasado, presente y futuro; futuro que depende de su identidad y capacidad para elegir una vía que lo proyecte como ser con sentido, con una existencia significativa y dueño de sus propias elecciones, mientras que su pasado se convierte en el rastro sobre el que asiste a su presente y construye futuras experiencias y significados. De esta manera, el desempeño ocupacional competente, realizante y la prospección ocupacional del sujeto ( proyecto de vida), esta supeditada a la capacidad para autorregular el comportamiento, el tiempo y sus actividades en su hacer cotidiano, a partir de la estructuración de esquemas de acción funcionales en la medida que respondan a las demandas ambientales y satisfagan las necesidades internas del sujeto, en términos de sobrevivencia, afecto, pertenencia, aprendizaje, creatividad y autorealización.

Complementado lo anterior, Chrystiansen (1999) plantea la dinámica identidad-ocupación-competencia y significado, como el eje sobre el cual el sujeto construye su historia y futuro, analizado desde el concepto de la identidad, la que asume como autoconocimiento e incluye el SELF con sus sentimientos, pensamientos y sensaciones, con el sentido de la capacidad para elegir e iniciar una acción que incluya el cuerpo y el pensamiento abstracto. Refiere que, para los Terapeutas Ocupacionales, la identidad se moldea partir de lo que se hace y cómo se hace, es decir, la ocupación participa en la construcción de la identidad, que lleva a ensayar la habilidad para participar en ocupaciones y mostrarse como persona competente, además ofrece un sentido de propósito, estructura el tiempo, posibilita darle significado a la vida y a su vez tener una vida significativa.

Esta identidad ocupacional, es una figura importante en la autonarración de la historia de vida, pues ofrece coherencia y significado a las experiencias diarias de la vida en sí misma, eventos evaluados de acuerdo al significado personal. Este significado de vida se deriva del contexto de identidad, que da visión de futuras posibilidades, donde cada quien espera el cumplimiento de sus metas a corto y largo plazo, que le den propósito a la existencia. La historia de vida, permite un panorama de la vida y se puede mostrar mediante 
un relato sistemático, que consta de eventos que suceden en progresiva secuencia. MC Adams (1997) refiere que construyendo e interpretando su historia de vida la persona muestra su identidad, se da sentido a sí mismo y a otros, ya que implica la expresión del yo a través de las acciones dentro del contexto, además ofrece una visión del constructo sobre el cual se puede o no edificar el propio proyecto personal. Es decir, la historia de vida y el significado de vida, se crea a través del hacer y el Selfing como el Yo que actúa, ya que cuando se vive con un propósito, se da un sentido y significado al proceso de vida. En relación con lo anterior, se considera que existe una relación importante entre identidad-ocupación-competencia y significado, sobre lo que el individuo construye sus metas y su propio proceso vital. Para Crystiansen (1999), las metas son imágenes posibles del YO, actúan como motivantes de la visión del YO y a su vez, de cómo se imagine en el futuro se establecen las metas. Por lo tanto, existe una estrecha relación entre las metas, el comportamiento motivado y el sistema de identidad.

En este sentido, el trabajo terapéutico involucra la construcción de identidad, ofreciendo vivencias que ayuden a la persona a explorar posibles sí mismos y obtener logros en sus acciones, de ahí que el profesional se apropie de la interacción que Christiansen propone : Identidad-Ocupación-Competencia-significado en los procesos de intervención, haciendo que dicha dinámica suceda, de manera que se reafirme el abordaje holístico de recuperación funcional y volicional del individuo. Dentro de esta perspectiva, la historia de vida se convierte en una herramienta importante en la evaluación y en el tratamiento, pero dándole un uso que ofrezca significado para el individuo, participe en la construcción de su identidad y de su proceso vital, integrando la ocupación y el hombre como un ser ocupacional por naturaleza, quien a partir de la acción significativa alcanza su desarrollo, su autorealización y trascende la experiencia vital. En este contexto, Viana Moldes I, García Pinto M.C y Ávila Álvarez A, Terapeutas Ocupacionales de la universidad de Coruña, en su artículo "Ocupación alienante Versus Ocupación significativa" (1999), afirman que el profesional de Terapia Ocupacional trabaja al individuo en el contexto de la percepción social de los significados de los valores que sustentan la ocupaciones para el ser humano y que por lo tanto, utiliza las ocupaciones significativas como medio de intervención para prevenir o minimizar la disfunción ocupacional, lo cual consiste en la oportunidad para el encuentro, el desencuentro y el reencuentro con uno mismo y con su entorno, a través de la apropiación del hacer comprometido, lo que beneficia la salud en la medida que aproxima y concretiza la expresión de los intereses, valores y destrezas del sujeto.

\section{Proyecto de Vida en personas CON DISCAPACIDAD MENTAL}

Para mí, quisiera que Dios me quisiera más, me gustaría quererme por fin, darme cuenta qué debo hacer conmigo, bañarme para empezar; vestirme, ser ordenado, aprender que las cosas son de otra manera. (Alberto, Usuario UPA Servitá 2003).

Voy a seguir yendo a terapias, quiero ser cajero de cabaré, tener una casa en Bogotá, casarme, tocar guitarra, tener una finca para sembrar, tener un avión, tener un carro. (Gustavo, Usuario UPA Servitá 2003).

Según el Ministerio de Salud en documento expedido en junio 18 de 1998, donde se presentan las últimas estadísticas sobre la incidencia de los trastornos mentales en Colombia, "la enfermedad mental, puede ser entendida como el resultado de expresiones biológicas, psicológicas y ambientales que se determinan mutuamente y que se configura dentro de lo que podría llamarse procesos de alteración en la integralidad del ser humano, que sobrepasa los tradicionales espacios en la psiquiatría y la psicopatología y se remonta a todos los factores y situaciones en los que se destaca un componente simbólico y comportamental, afectando las condiciones de vida de las personas, su familia y la sociedad". 
En este sentido, para el Terapeuta Ocupacional la salud mental del individuo es un componente vital de adaptación, implica la capacidad adaptativa que posibilita al individuo adquirir y desempeñar sus roles ocupacionales (autocuidado, productivo y de esparcimiento ), tener una pertenencia familiar, social y laboral dentro de una interacción dinámica que satisfaga sus expectativas internas y externas, así como proyectarse hacia la autorealización. Cuando aparece la enfermedad mental o bien el trastorno, la interacción se altera o pierde manifestándose en una disfunción psicosocial y por ende ocupacional lo que conlleva progresivo aislamiento familiar, laboral y social del individuo, con la pérdida de motivación intrínseca para interactuar con su entorno y participar en ocupaciones que le brinden bienestar y significado.

Frankl (1990), afirma que la gente vive hoy un vacío existencial y que éste se manifiesta sobre todo en el aburrimiento. Son numerosos los pacientes que acuden a la consulta con la sensación de vacío interior o "vacío existencial" con la sensación de un absurdo radical de su existencia y la pérdida del sentido de vida. Sentimientos presentes no sólo en lo que él llama la Tríada Neurótica (depresión, agresión y adicción), sino también en personas víctimas impotentes de una situación desesperada, que no se puede cambiar, donde sólo se puede modificar la propia actitud, cambiarse a sí mismo, madurando, creciendo, trascendiendo y dando así testimonio de la facultad más humana del hombre: la de transmutar una tragedia personal en triunfo. Este sentimiento de falta de sentido va en aumento; cada vez son más las personas que buscan en vano el sentido de la vida. Muchas publicaciones, basadas en los test logoterapéuticos, atestiguan el incremento del sentimiento de absurdo de la vida en todo el mundo. Por su parte Miller en 1990, describe la experiencia de transformación de individuos con enfermedades psiquiátricas crónicas, lo que refirió como crisis espiritual y la describió tomando a Oates (1978) así : estado en el que la persona esta agudamente enferma y con gran significación e interpretación de caos a su existencia.
Desde otra perspectiva, este caos de la existencia en una persona con discapacidad mental, le causa efectos en su desempeño ocupacional, ya que se interrumpe su proceso vital y sus expectativas de vida deben ser ajustadas a su nueva condición. Una de las mayores consecuencias se centra en el hecho de que las relaciones con el entorno se deterioran o bien se empobrecen, dificultando esto la adaptación a su nuevo estilo de vida. Es así, como la capacidad de prospección y elaboración de el proyecto de vida en la persona con discapacidad mental, se encuentra quebrantada, por el mismo proceso de la enfermedad que lleva a la pérdida de oportunidades, por efectos de las crisis, la falta de apoyo de su núcleo familiar o por la insuficiente planeación estatal para la capacitación y ubicación laboral de este tipo de población ; de otra parte, en las personas con enfermedad mental se refleja un déficit adaptativo en su desempeño ocupacional competente, ya que en ellas se encuentran las siguientes características en sus funciones Yoicas: pobre funcionamiento del Yo, con dificultades en el manejo de la realidad, en la capacidad de juicio, en el autocontrol, en las relaciones objetales, en el autoconocimiento y la autoestima; alteración en sus procesos de pensamiento, en el manejo de estímulos ambientales, en el manejo del estrés, en la capacidad para integrar experiencias, pobreza en su función autónoma y en las de dominio y competencia. Estas alteraciones se comportan de diferente manera según sea el tipo y grado del trastorno que presente el individuo, pero que de manera importante afectan su capacidad para proyectarse, para diseñar su proceso vital y establecer estrategias para desarrollarlo, de ahí que su sentimiento de autorealización y su sentido de vida no ocurran según el potencial de la condición humana. A continuación se mencionan las dificultades del desempeño ocupacional identificadas a partir de la experiencia directa y analizadas con base en el Modelo de la Ocupación Humana y del Desempeño Ocupacional Realizante :

1. Distorsiones en el campo volicional con limitada o ausente motivación interna para participar e idear 
actividades placenteras, gratificantes, productivas y que ofrezcan nuevas experiencias, de manera que el actuar ocupacional lo lleve a su autorealización.

2. Alteraciones en la causación personal, donde las dificultades a nivel de identidad y autoestima, determinan que la persona no logre reconocer sus capacidades y necesidades, y proyectar, organizar y seleccionar sus procesos ocupacionales.

3. Además, esta pobre identidad del Yo, ocasiona dificultad para identificar, ejecutar y explorar intereses y metas.

4. Esta pobreza en los componentes de identidad y autoestima, sumada a sus alteraciones en el área de los intereses ocupacionales, lleva a que estas personas no logren identificar objetivos y metas a corto, mediano y largo plazo, con consecuencias en su prospección ocupacional, la que presenta alteraciones en cuanto a que no existe o bien se presenta de forma distorsionada, no acorde a su realidad, edad, estatus, rol ocupacional, nivel de competencia y recursos.

5. La estructura de valores y creencias, está supeditada a los valores y creencias sobre un sí mismo incapaz, además se presenta una restricción en dicha escala limitándose a direccionarla sobre lo que maneja su entorno familiar, o bien distorsionados por procesos de pensamiento irracionales. En esto influyen los patrones y creencias culturales ya que, no estimulan el actuar ocupacional o bien descalifican la realización individual de actividades.

6. Las limitaciones que la patología le impone a sus competencias ocupacionales, ocasiona dificultad para la realización independiente de las actividades básicas de la vida diaria, para establecer relaciones significativas íntimas y amistosas dentro de un marco de reciprocidad, para organizar su comportamiento ocupacional en hábitos y rutinas, para utilizar la cognición en el planeamiento y secuenciamiento de sus ocupaciones diarias, y en relación con éstas, solucionar problemas. Es decir, la discapacidad mental ocasiona distorsión en los procesos humanos, ya que la persona encuentra obstáculos para el desarrollo de su potencial, para encontrar significado en las experiencias y satisfacer sus necesidades de subsistencia, socialización, dominio y actualización.

7. Dificultades para desarrollar procesos ocupacionales con significado y direccionalidad para satisfacer sus necesidades de vestido, alimentación y vivienda de manera autónoma, identificando dichas necesidades y estableciendo estrategias para solucionarlas dentro de un contexto real y con base en sus intereses, capacidades, valores y creencias.

8. Pobre motivación, dificultad para solicitar ayuda y utilizar los recursos comunitarios, actuando de manera aislada o en contravía con su entorno.

9. Vulnerabilidad extrema al estrés, con la aparición de crisis que alteran sus procesos personales y ocupacionales, afectando la continuidad de su proceso vital, el bienestar de sí mismo y su familia.

10.Aparición de procesos de deterioro en sus habilidades sensorio-motoras, cognitivas, psicosociales y espirituales, en la medida que no vive experiencias nuevas y dadas por el curso de su enfermedad. Aquí el contexto participa en cuanto ofrece limitadas oportunidades de exploración y creación, además por la falta de sensibilización social ante este tipo de discapacidad.

11.Dificultad para identificar sus roles y desempeñarlos de manera independiente de acuerdo a su estatus de edad, encontrándose la resignación a vivir bajo el rol de enfermo y careciendo de motivaciones para explorar nuevas experiencias y así asumir un cambio en dichos patrones de acción.

En este sentido, las personas con discapacidad mental, tienen una gran variedad de dificultades, que limitan su evolución en propósitos ascendentes como la organización, la integración, la productividad y la realización, lo que afecta su sentimiento de competencia y bienestar, y además, aporta pobre conciencia sobre la importancia para su salud, como el tener un proyecto de vida, conocer los servicios que ofrece su 


\section{ARTÍCULOS INÉDITOS}

comunidad y estrategias para acceder a ellos como forma de lograr sus metas personales.

Frente a la problemática planteada, el Terapeuta Ocupacional tiene como herramienta fundamental de trabajo, el manejo de la actividad como medio que potencializa el desempeño ocupacional del individuo con discapacidad al igual que su sí mismo (representado por los objetos que manipula) y el entorno, quienes integrados en la acción, actúan como puente que comunica el mundo interno y externo de la persona dándole un sentido unificado de sí mismo. De esta manera, se busca que a través de la exploración y la manipulación del sí mismo y los objetos en los procesos del hacer, el individuo logre resignificar su existencia, siendo funcional y productivo. Para esto, la actividad debe actuar sobre sus dimensiones sensoriales, motoras, perceptuales, cognoscitivas, psicológicas, sociales y espirituales, de manera que se alimente la interdependencia existente entre estas y así se aborde al ser único e integral.

Además, el hacer trasciende cuando ofrece propósito, da placer y significado a la existencia del que lo realiza, lo que se logra cuando el sujeto vivencia que su acción produzca un cambio en su interior, en los objetos y en su entorno inmediato. Por lo tanto, la utilización de objetos que movilicen canales afectivos en el sujeto facilitan la resignificación de las relaciones con sí mismo, los demás y por ende de su misma discapacidad, aspectos que en la medida de lo posible motivan hacia la recuperación de la funcionalidad o bien de nuevas búsquedas por parte del sujeto. En este orden de ideas, para que la resignificación y las búsquedas se den en el interior del sujeto y se cristalicen en metas y propósitos dentro de un proyecto de vida, se propone trabajar desde la dimensión espiritual de la ocupación y el ser humano (Santacruz, Enfoque de la Logoterapia, 2002), visión compartida con Alicia Trujillo (2002), quien la contextualiza en su libro "Terapia Ocupacional conocimiento y práctica en Colombia", afirmando que la dimensión espiritual del desempeño ocupacional trasciende el dominio del hacer y ejecutar, y se refiere a la sensibilidad de las personas por las fuentes más esenciales de motivación y la búsqueda de propósito en la vida. La espiritualidad tiene que ver con las creencias fundamentales de la persona sobre la naturaleza y el sentido de vivir; una persona puede sentirse en sintonía espiritual y reflexionar sobre su proyecto de vida cuando se involucra en diversas actividades tales como escuchar música, observar una puesta de sol, escribir un poema, hacer reminiscencias familiares o participar en proyectos solidarios o de alto contenido social (Hettinger, 1996, referido por Trujillo 2002).

El concepto de la espiritualidad ha tomado un importante lugar en el modelo de práctica desarrollado por la Canadian Association of Occupational Therapys (CAOT,1991), así como Belligham Et. col en 1989, quienes describieron una manera específica para establecer y mantener la dimensión espiritual, trabajando la conexión de las tres esferas espirituales : conexión con el sí mismo, conexión con los otros y conexión con la existencia y el todo ; a partir de las cuales se pretende ligar estrategias desde la perspectiva de Terapia Ocupacional de manera que se logren aspectos de autonomía personal y social en el cliente (Enfoque de la Logoterapia, Santacruz, 2002). Un proceso de intervención integral desde la espiritualidad del sujeto y la ocupación, implica la habilidad del terapeuta para interpretar la importancia de las actividades en concordancia con la realidad del cliente y consistentes con sus metas personales.

Para el abordaje de esta temática, como es la situación del proyecto de vida en personas con discapacidad mental se proponen las siguientes estrategias de intervención: en primera instancia, talleres psicoeducativos con la población con discapacidad, con el fin que cada uno diseñe su proyecto de vida, mediante actividades soportadas en los principios de la Terapia Ocupacional y los enfoques teóricos de la Logoterapia, la Psicodinamia y la aproximación cognitivo-conductual. En segundo lugar, actividades bajo un modelo de trabajo grupal, donde el colectivo se constituye en el núcleo de la intervención haciendo énfasis en los aspectos subjetivos del individuo y en la propuesta de 
una actividad con propósito y estructurada sobre sus características, intereses y fines de la temática, esto implica la acción instrospectiva, creativa, lúdica y relacional, dentro de un espacio cognoscente y socioemocional que lleve a la internalización de la temática en cuestión. Por último, asesoría a la familia, es una estrategia que parte del reconocimiento del papel fundamental de la familia para liderar la propuesta de vida de su pariente, la cual requiere información y soporte permanente, atención que se da mediante la visita domiciliaria.

Los temas trabajados dentro de las estrategias planteadas anteriormente, son estructurados a partir de lo que plantea el Enfoque de la Logoterapia (Santacruz 2002), donde se desarrollan las tres esferas propuestas por Bellingham, Cohen, Jones and Le Roy, (1989), articulándolas con la esencia y fin del presente proyecto, con los principios existenciales de la vida y el ser humano y con las características de la población.

\section{PRIMERA ESFERA - CONEXIÓN CON EL SÍ MISMO :}

- Sentido de sí mismo

- Yo real Versus Yo ideal

- Sentido de vida

- Identidad y autoimagen

- Valores y creencias

- Intereses

- Historia de vida

- Perfil ocupacional

- Autonomía y toma de decisiones

SEGUNDA ESFERA - CONEXIÓN CON LOS OTROS :

- Sistema relacional yo-mundo

- Sentido de sí mismo dentro del entorno familiar

- Participación de la familia en el proceso vital

- Identidad y familia

- Exploración familiar de sus fortalezas y debilidades

- Historia de vida y Familia

- Proyecto de vida y familia

\section{TERCERA ESFERA - CONEXIÓN CON LA}

\section{CREACION Y PROPÓSITO DE VIDA :}

- Sentido de mundo y vida al futuro

- Sentido existencial

- Proyección del sí mismo a corto, mediano y largo plazo

- Elaboración de metas a corto, mediano y largo plazo

- Diseño de estrategias para el logro de metas

- Proyecto de vida y toma de decisiones frente a éste

- Elaboración hoja de vida

- Experiencias de búsqueda de oportunidades laborales

- Asumir actitudes con compromiso

\section{Conclusiones}

1. La atención a población con discapacidad mental y cognosciitiva tiene como marco desarrollos legislativos que, si bien han logrado avances en ciertos aspectos, no han conducido aún a procesos coordinados y eficientes en cuanto a cobertura, integración de los servicios que se prestan, prevención de la discapacidad y apoyo al entorno social del discapacitado. La legislación debe propender por el logro de ambientes favorables para la participación familiar, escolar, laboral, recreativa y de acceso de las personas con discapacidad a todos los servicios, en igualdad de condiciones a las de otros ciudadanos ( Trujillo 2002).

2. La persona con discapacidad mental debe ser educada y capacitada en estrategias que favorezcan visualizar y diseñar su proyecto de vida y de manera importante, ser orientado para utilizar sus recursos personales y los que ofrece su comunidad; esto debe proyectarse a la familia ya que ésta se considera como factor de apoyo fundamental para que sus miembros logren obtener también sentido de vida.

3. La persona con discapacidad mental, requiere del apoyo de su contexto familiar y social, para lograr apropiarse de sus recursos, aspecto que 
tiene influencia cultural en la medida que existe un estigma hacia la enfermedad mental que lleva a que los sujetos funcionales consideren al enfermo no apto para vivir en comunidad ; de ahí la importancia de proyectos psicoeducativos tanto para la persona con discapacidad como para la familia y la comunidad.

4. La realización de proyectos como el presente se hacen urgentes en la medida que la persona con discapacidad mental tiene el derecho de tener y cumplir un proyecto de vida como ser humano, con un sentido de sí mismo, de vida y del todo, de manera que esta posibilidad revierta en su bienestar e inclusión social.

5. Se evidencian dificultades importantes en este tipo de población en cuanto a su sentido de pertenencia y prospección ocupacional, corroborando lo presentado anteriormente sobre su capacidad para diseñar el proyecto de vida, diferenciándose claramente que cuando prevalece la problemática mental, la limitante se da básicamente por su pobre motivación intrínseca y su baja autoestima, mientras que en los sujetos donde es mayor el compromiso cognitivo, este factor no da la posibilidad de abstraer lo que concierne al concepto, sus metas y estrategias. De esta manera, su sentido de sí mismo es pobre o distorsionado su sentido de vida ofrece un panorama de vacío existencial donde su significado de vida no es claro y sobre éste pesa los pensamientos negativos sobre sí mismo y el futuro, la conciencia de enfermedad participa profundamente; así mismo, su propósito de vida casi es inexistente ya que encuentra un sentimiento de desesperanza que lucha con el deseo de sanar y actuar libremente y con autonomía.

6. El Terapeuta Ocupacional como profesional que trabaja con la naturaleza ocupacional del hombre y su búsqueda de autorealización, está en la capacidad de abordar procesos vitales estructurados en el proyecto de vida, como herramienta que subsane el vacío existencial que la discapacidad mental genera en el sujeto que la padece.
7. La calidad de la alianza terapéutica se convierte en la habilidad fundamental que debe poseer el Terapeuta Ocupacional para garantizar la calidad de la relación con los usuarios y de manera directa la calidad del servicio prestado. Así mismo, la calidad del servicio está dado por la pericia del terapeuta para seleccionar, diseñar y graduar una serie de actividades estructuradas a partir del conocimiento de la población y la identificación de sus necesidades e intereses.

8. Trabajar el proyecto de vida desde el enfoque de la Logoterapia es un reto para el Terapeuta Ocupacional, ya que generar sentido de vida en alguien que siente que es un derecho que se le ha negado, alguien cuyos recursos materiales no le posibilitan grandes proyecciones y que pertenece a una sociedad que está en crisis tanto de valores como de oportunidades, lleva a cuestionar hasta dónde se puede intervenir desde la ocupación para la ocupación, si el entorno no la está potencializando. En esta medida donde quedan los esfuerzos?

Alberto: unos pierden oportunidad, otros se encuentran con mucha preocupación u otros como la mayoría, si encajan en su ambiente apropiado (Usuario UPA Servitá. 2003)

Hugo : Quién soy? un conquistador de Colombia $y$ del universo. Qué piensan que soy yo? mente, espíritu, deportista, viajero, vendedor; administrador de Colombia. (Usuario UPA Servitá 2003)

\section{Referencias Bibliográficas}

BUNSON Matthew. 1997. La sabiduría del Dalai Lama. Publicado bajo licencia de Dutton Plume. España.

COOK J. 1994. Innovation and leadership in a mental health facility. The American Journal of Occupational therapy.49(7).

CHARLES H. CHRISTIANSEN, 1999. Defining lives : occupacion as identity an essay on competence, coherence and creation of meaning. American Journal of Occupational Therapy. 53 (6).

DUNCAN E. 1999. Occupational therapy in mental health : its time to recognise that it has come age. British journal of occupational therapy. 62 (11). 
EGAN M., DELAAT M. 1997. The Implicit spirituality of occupational therapy practice. Canadian journal of Occupational therapy. 64(3).

EGAN M., DELAAT M. 1994. Considering spirituality in occupational therapy practice. Canadian journal of Occupational therapy. 61(2).

ETCHEVERRY J,A. 1999. Salud mental, Logoterapia y liberación. Editorial Lugar. Buenos Aires.

FINLAYSON, M, EDWARDS, J. 1995. Integrating the concepts of health promotion and comunity into occupational therapy practice. Canadian Journal of Ocupational therapy. 62(2). Pag. 76-80.

FRANCO Zoila. 1994. Desarrollo Humano y de valores en salud. Instituto Colombiano para el fomento de la educación superior. Bogotá.

FRANKL Victor. 1990. El hombre doliente. Editorial Herder. Madrid.

FRANKL Victor. 1990. Psicoterapia al alcance de todos. Editorial Herder. Madrid.

GROSSMAN J. 1990. A prevention model for occupational therapy. The american journal of occupational therapy.45(1).

KAUTZMAN L. 1995. Alternatives to psychosocial fielwork : Part of the solution or part of the problem. AOTA. American Journal of Occupational Therapy. 49.( 1).

LAMPERT R. 1998. Occupation and lifestyle: implications for mental health practice. British journal of occupational therapy. 61(5).

MEESON Beverly. 1998. Occupational therapy in comunity mental health, part 1: intervention choice. British journal of Occupational Therapy. 61(1).7-11.

MEESON Beverly. 1998. Occupational therapy in comunity mental health, part 2: Factors influencing intervention choice. British journal of Occupational Therapy. 61(2). 5761.

MINISTERIO DE SALUD. 1.998. Política Nacional en Salud Mental. Art. 77 del decreto 1292 de 1.994.

PARDO Inés. 1999. Jóvenes construyendo su proyecto de vida. Editorial aula abierta Magisterio. Bogotá.

POLONIO B, DURANTE M, NOYA B. 2001. Conceptos fundamentales de Terapia Ocupacional. Editorial Panamericana Medica. Madrid.

SANTACRUZ Marta y cols. 2003. Proyecto de vida y competencia ocupacional en adolescentes y adultos con discapacidad mental y cognoscitiva. Innédito. Institución Universitaria Fundación Escuela Colombiana de Rehabilitación. Bogotá.

SANTACRUZ Marta y cols. 2002 Promoción de patrones ocupacionales saludables en población adulta con discapacidad mental. Innédito. Institución Universitaria Fundación Escuela Colombiana de Rehabilitación. Bogotá.

SANTACRUZ Marta y cols. 2002. Promoción del comportamiento ocupacional competente a partir del conocimiento de sus deberes y derechos en población con discapacidad cognitiva y mental, dentro y fuera de su entorno familiar. Innédito . Institución Universitaria Fundación Escuela Colombiana de Rehabilitación. Bogotá.
SANTACRUZ M. 2002. Enfoque de la Logoterapia. Institución Universitaria Fundación Escuela Colombiana de Rehabilitación. Innédito. Bogotá.

SANTACRUZ M. 2001. Enfoque Cognoscitivo-conductual. Institución Universitaria Fundación Escuela Colombiana de Rehabilitación. Innédito. Bogotá.

SAVATER Fernando. 1999. Las preguntas de la vida. Editorial Ariel. Barcelona. España.

SAVATER Fernando. 1993. Diccionario Filosófico. Editorial Planeta. Barcelona España.

SECRETARIA DISTRITAL DE SALUD DE BOGOTÁ. 2001. Lineamientos de Atención en salud para las personas con discapacidad en Bogotá. Alcaldía Mayor de Bogotá

SECRETARIA DISTRITAL DE SALUD DE BOGOTÁ. 2001. Plan Distrital de Discapacidad, 2001-2005. Alcaldía Mayor de Bogotá.

REBEIRO K. 1998. Occupation as means to mental health. Canadian Journal of occupational therapy. 65(1).

TAYLOR A, RUBIN R. 1999. How do occupational therappist define: their role in a comunity mental health setting? British journal of occupational therapy. 62 (2).

TRUJILLO Alicia. 2002. Terapia Ocupacional, conocimiento y práctica en Colombia. Universidad Nacional de Colombia Sede Bogotá.

TRUJILLO Alicia. 1995. Desempeño ocupacional realizante : un modelo sobre los propósitos de la Terapia Ocupacional. Revista de la Ocupación Humana. Asociación Colombiana de Terapia Ocupacional. Bogotá. 6(2).11-21.

URBANOWSKI R., VARGO J. 1994. Spirituality, daily practice, and the occupational performance model. Canadian journal of Occupational Therapy. 61(2).

WEILL Peter. 1997. Los límites del ser humano. Editorial los libros de la liebre de marzo. Barcelona. España. 